\title{
Radical Hysterectomy With and Without Neoadjuvant Chemotherapy in Patients With Cervical Cancer Stage IB-IIB
}

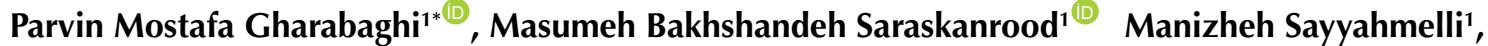 \\ Mehri Jafari ${ }^{1}$, Elahe Saheb Olad Madarek ${ }^{1}$, Maryam Vaezi ${ }^{1}$, Vahideh Rahmani ${ }^{1}$, Ali Adili ${ }^{1}$, Malahat \\ Ebrahimpour ${ }^{1}$, Atie Amidfar ${ }^{1}$, Maryam Pourbargi ${ }^{1}$
}

\begin{abstract}
Objectives: In this study, radical hysterectomy, followed by neoadjuvant chemotherapy (NACT) in patients with locally advanced cervical cancer (LACC) was compared with radical hysterectomy in patients with early-stage cervical cancer.

Material and Methods: This retrospective comparative observational study was performed on 13 patients with LACC International Federation of Gynecology and Obstetrics (FIGO) stage IB2-IIB who underwent a radical hysterectomy after NACT between March 2014 and November 2018. This group was compared with 18 patients undergoing radical hysterectomy with cervical cancer FIGO stage IIA-IB1 in the same period of time.

Results: In the NACT group, 8 (61.5\%) and $5(38.4 \%)$ patients were in stages IIB and IB2, respectively, and $13(72.2 \%)$ cases were in the IB1 stage in the non-NACT group. Post-operative blood transfusion in the NACT group was significantly higher compared to the non-NACT group [5 $(38.4 \%)$ patients versus $0, P=0.008$ ]. The estimated blood loss (EBL) and operative time were similar between the groups. Finally, there were no significant differences in terms of intra-operative and other post-operative complications.

Conclusions: Radical hysterectomy after NACT in women with LACC seems to be safe and reduces the need for radiation in patients with NACT who are at stage IIB. These results need to be confirmed in studies with a larger patient sample.

Keywords: Abdominal radical hysterectomy, Cervical cancer, Neoadjuvant chemotherapy, Complications
\end{abstract}

\section{Introduction}

Cervical cancer is a global health problem for women. Despite the availability of vaccination and screening, it remains the fourth most common cancer in women. Approximately $85 \%$ of cases occur in developing countries (1). The age distribution of this type of cancer shows three peaks at ages 35,50 , and 70 (2), which is one of the main causes of death from cancer (3).

The stage of cervical cancer is the most important factor in its prognosis. The of the uterine cervix cancer has traditionally been clinically staged although surgical and radiologic evaluations are now part of the assigning stage (4-6) Surgical and radiologic staging provide important information that can impact treatment (7) However, the International Federation of Gynecology and Obstetrics (FIGO) has demonstrated that clinical staging is preferable for several reasons. It is more accessible for low-resource settings, in which cervical cancer remains the most common malignancy among women. Likewise, it may be better for assessing locally advanced disease (i.e., tumor size, vaginal and parametrial involvement) and it avoids surgery in women who are not candidates for surgical treatment (8) In the FIGO classification system for cervical cancer staging, stage IB is divided into IB1 and IB2 subgroups based on the tumor size, which has been recognized as an important prognostic factor in poor outcomes for stage IB cervical cancer, correlating with the tumor recurrence rate and decreasing survival (9) Other important factors in the prognosis of cervical cancer include parametrial involvement, lymphovascular space invasion, and pelvic lymph nodal status (9).

Surgery is the standard treatment for stage IA-IB1 cervical cancer (1). However, early surgery is not recommended in women with locally advanced cervical cancer (IB2-IVA) because recovery is not frequently achieved and the need for adjuvant therapy would be the next alternative in this regard. Chemoradiation is the main treatment in patients with stage IB2-IIB and its profits will be higher for them.

Radiotherapy and chemoradiation can cause long-term treatment-related complications and serious problems for patients, especially young patients with sexual activity. Because most patients are young and life expectancy 
Key Messages

- Neoadjuvant chemotherapy in the treatment of cervical cancer reduces the need for radiotherapy and its side effects.

is high in this age group, and the quality of life is an important issue for them in addition to survival (9).

Neoadjuvant chemotherapy (NACT), followed by radical hysterectomy is a completely different treatment which seems to have some special advantages over chemoradiation, including micrometastasis elimination, lower toxicity, easier patient management, tumor size reduction, and decreases in the risk of positive lymph nodes and the need for radiotherapy. It also increases the possibility of surgery and makes the tumor more sensitive to subsequent radiation if necessary $(1,9)$.

Given the above-mentioned explanation, this study evaluated the safety and morbidity of radical hysterectomy in women with LACC after platinum-based NACT.

\section{Materials and Methods}

After obtaining the approval of the Institutional Review Board, the current retrospective comparative observational study was performed at the Gynecologic Department of Al-Zahra hospital of Tabriz University of Medical Sciences. All patients undergoing radical hysterectomy between March 2014 and November 2018 were eligible for inclusion in this study.

The inclusion criteria included patients with cervical cancer (squamous cell carcinoma [SCC], adenocarcinoma, adenosquamous carcinoma, clear cell carcinoma, and the like) based on pathologic reports, IB-IIB stage based on FIGO staging system, no other malignancy in patients, a full record of required information in the fil, and a willingness to participate in the study.

On the other hand, the exclusion criteria were patients not being in the IB-IIB stage according to the FIGO staging system, lack of required information in the patient's records, cervical cancer along with other cancers, and an unwillingness to participate in the study.

All patients underwent examination under general anesthesia, staging based on the FIGO staging system, cystoscopy, and chest, along with abdominal and pelvic computed tomography prior to radical hysterectomy. Of the 31 patients enrolled in the study, 13 cases received NACT, and their mean age and median age were 39.9 and 41, respectively. Eight patients were at stage IIB and 5 of them were at stage IB2. Eighteen patients had radical hysterectomy initially, and their mean age and median age were 52.6 and 47 , respectively. Thirteen cases were at stage IB1 while two and three other cases were at stages IB2 and IIA, respectively.

In the NACT group, patients were given 3-6 cycles of cisplatin $50 \mathrm{mg} / \mathrm{m}^{2}$ and taxol $175 \mathrm{mg} / \mathrm{m}^{2}$ every 3 weeks, and the liver and kidney test was requested before each injection complete blood count. For patients with an interval of 2-6 weeks after the last chemotherapy, radical hysterectomy and pelvic lymph node dissection was performed.

Charts were abstracted, and the analyzed data included intra-operative complication, final histology diagnosis, post-operative complications, and the need for adjuvant treatment.

The amount of blood lost during the surgery, hemoglobin $(\mathrm{Hb})$ and hematocrit levels before and after the surgery, and the duration of surgery were recorded in patient records.

Wound infection is defined as purulent discharge from the wound, redness, warmth, and tenderness of the stance. In addition, wound dehiscence is defined as the opening of surgical sutures with serous discharge, and urinary system damage is defined as penetrating trauma to this structure in the surgery.

The operating time was defined from the beginning of skin incision to the completion of skin closure. The estimated blood loss (EBL) was calculated by considering the difference in the total amounts of suctioned and irrigation fluids and counting the number of bloody and long gauzes.

After the surgery and based on the final pathology of radical hysterectomy, some patients received adjuvant treatment, which included radiotherapy, chemoradiation, and/or chemotherapy.

Patients were followed by pelvic exams every three months to two years and then every six months to three years and then annually.

Shapiro-Wilk test was used to evaluate the normal distribution of the data of the collected variables. Moreover, frequency and percentage, as well as mean and standard deviation were applied for categorical and continuous variables, respectively.

Categorical variables among the groups (NACT vs. nonNACT) were compared by using a $\chi^{2}$-based proportion test as implemented in the Fisher's exact test. A twotailed $t$ test (when the normality and homogeneity of variance assumptions were met) or the non-parametric Mann-Whitney U test was used for continuous variables. In all statistical tests, a confidence interval (CI) of 95\% and $\mathrm{P}<0.05$ were considered as significant differences. Eventually, the statistical analysis was performed using the IBM SPSS program, version 26.0.

\section{Results}

Patients' Characteristics

Thirteen patients with LACC undergoing laparotomy and radical hysterectomy after NACT were compared with 18 patients with early stage cervical cancer who underwent laparotomy and radical hysterectomy. FIGO staging was different between the two groups. In the NACT group, 8 (61.5) and 5 (38.4) patients were in stages IIB and IB2, 
respectively, and 13 (72.2) cases were in the IB1 stage in the non-NACT group. The mean $\mathrm{Hb}$ before the operation was $11.79 \mathrm{~g} / \mathrm{dL}$ and $13.53 \mathrm{~g} / \mathrm{dL}$ in the NACT and nonNACT groups, respectively (Table 1).

Intraoperative Characteristics

Intra-operative characteristics are provided in Table 2. The mean operating time and EBL) were similar in both groups. Intra-operative complications were recorded as well. Three $(23 \%)$ patients in the NACT group and $3(16.6 \%)$ patients in the non-NACT group had intraoperative blood transfusion thus the rate of post-operative $\mathrm{Hb}$ loss was not compared in the two groups. One patient in the non-NACT group had a ureteral injury, which was diagnosed during the operation and ureteroneocystostomy was performed accordingly.

\section{Pathology Data}

In the NACT group, $10(76.9 \%)$ and $2(15.3 \%)$ patients were SCC and adenocarcinoma, respectively, and 1 (\%7.6) patient was clear cell carcinoma. In the non-NACT group, $15(83.3 \%)$ and $3(16.6 \%)$ patients were SCC and adenocarcinoma, respectively. Table 3 presents final pathologic characteristics (i.e., positive pelvic lymph node, positive margin, and lymphatic vascular space invasion), indicating that there was no significant difference between the two groups.

Table 1. Baseline Patients' Characteristics

\begin{tabular}{lccl}
\hline Variable & $\begin{array}{l}\text { NACT } \\
(\mathbf{n = 1 3})\end{array}$ & $\begin{array}{l}\text { Non-NACT } \\
(\mathbf{n = 1 8})\end{array}$ & $\boldsymbol{P}$ Value \\
\hline \multicolumn{4}{c}{ Continuous, Mean (SD) } \\
\hline Age (year) & $39.9(10.06)$ & $49.7(9.09)$ & 0.008 \\
$\mathrm{Hb}(\mathrm{gr} / \mathrm{dL})$ & $11.8(0.8)$ & $13.53(1.1)$ & 0.00 \\
\hline \multicolumn{4}{c}{ Categorical, No. (\%) } \\
\hline FIGO stage & 0 & $13(72.2)$ & 0.00 \\
IB1 & $5(38.4)$ & $2(11.1)$ & 0.099 \\
IIA & 0 & $3(16.6)$ & 0.245 \\
IIB & $8(61.5)$ & 0 & 0.00 \\
\hline
\end{tabular}

Note. NACT: Neoadjuvant chemotherapy; SD: Standard deviation; FIGO: International Federation of Gynecology and Obstetrics.

Table 2. Intra-operative Patients' Characteristics

\begin{tabular}{lccc}
\hline Variable & $\begin{array}{c}\text { NACT } \\
(\mathbf{n = 1 3})\end{array}$ & $\begin{array}{c}\text { Non-NACT } \\
(\mathbf{n = 1 8})\end{array}$ & P Value \\
\hline \multicolumn{4}{c}{ Continuous, Mean (SD) } \\
\hline Operative time (min) & $323(123.5)$ & $333.3(171.4)$ & 0.936 \\
Estimated blood loss (cc) & $265.3(36.8)$ & $281.3(56.2)$ & 0.159 \\
\hline \multicolumn{5}{c}{ Categorical, No. (\%) } \\
\hline Intra-operative complications & $3(23)$ & $3(16.6)$ & 0.676 \\
Intra-operative blood transfusion & 0 & 0 & \\
Nerve injury & 0 & 0 & 1 \\
Intestinal injury & 0 & $1(5 / 5)$ & \\
Ureteral injury &
\end{tabular}

Note. NACT: Neoadjuvant chemotherapy; SD: Standard deviation.
Postoperative Complication

Data on postoperative complications are indicated in Table 4. Blood transfusion in the NACT group was significantly higher compared to the non-NACT group [5 (38.4\%) patients versus $0, P=0.008]$. Based on the results, 3 $(25 \%)$ and $2(8.1 \%)$ patients in the NACT and non-NACT groups had infection/dehiscence at the site of surgery, respectively, although the difference was not statistically significant. One (7.6\%) patient in the NACT group needed re-laparotomy the next day due to a drop in $\mathrm{Hb}$ and anuria. Although no active bleeding was observed during the operation, there was a massive hemoperitoneum. The patient died 10 days after the first laparotomy with disseminated intravascular coagulation (DIC) and endorgan damage.

\section{Adjuvant Therapy}

Table 5 presents post-operative adjuvant therapy. In the NACT group, 4 (33.3) and 3 (25\%) patients received chemoradiation and chemotherapy, respectively, one $(8.3 \%)$ patient received radiation while 4 (33.3\%) patients, all of whom were stage IIB, required no adjuvant therapy. In the non-NACT group, 11 (61.1\%) patients underwent chemoradiation and $1(5.5 \%)$ patient received chemotherapy. Based on the findings, 6 (33.3\%) patients required no additional treatment. However, the differences between the two groups were not statistically significant and the final pathology of $5(38.4 \%)$ patients in the NACT group was no residual tumor (Figures 1 and 2).

Follow-up

The median follow-up in non-NACT and NACT patients

Table 3. Pathologic Data

\begin{tabular}{llll}
\hline Variable & $\begin{array}{l}\text { NACT } \\
(\mathbf{n = 1 3})\end{array}$ & $\begin{array}{l}\text { Non-NACT } \\
(\mathbf{n = 1 8})\end{array}$ & P Value \\
\hline \multicolumn{4}{c}{ Continuous, Mean (SD) } \\
\hline Follow-up, median (range) & $33(12-43)$ & $31(14-69)$ \\
\hline \multicolumn{4}{c}{ Categorical, No. (\%) } \\
\hline Positive margin & 0 & $1(5.5)$ & 1 \\
Positive pelvic nodes & $3(23)$ & $6(33.3)$ & 0.696 \\
Patients without relapse & $3(23)$ & $8(44.4)$ & 0.275 \\
Patients alive & $11(91.6)^{*}$ & $15(83.3)$ & 0.632 \\
\hline
\end{tabular}

Note. NACT: Neoadjuvant chemotherapy; SD: Standard deviation; LVSI: Lymphatic vascular space invasion.

Table 4. Post-operative Complications

\begin{tabular}{llll}
\hline Variable & $\begin{array}{l}\text { NACT } \\
(\mathbf{n = 1 3})\end{array}$ & $\begin{array}{l}\text { Non-NACT } \\
(\mathbf{n = 1 8})\end{array}$ & $\begin{array}{l}\boldsymbol{P} \\
\text { Value }\end{array}$ \\
\hline \multicolumn{4}{c}{ Categorical, No. $(\%)$} \\
\hline Post-operative blood transfusion & $5(38.4)$ & 0 & 0.008 \\
Wound infection/dehiscence & $3(25)$ & $2(11.1)$ & 0.364 \\
DIC/Reoperation & $1(7.6)$ & 0 & 0.419 \\
\hline
\end{tabular}

Note. NACT: Neoadjuvant chemotherapy; DIC: Disseminated intravascular coagulation. 
Table 5. Adjuvant Therapy

\begin{tabular}{lccc}
\hline Variable & $\begin{array}{c}\text { NACT } \\
(\mathbf{n = 1 3})\end{array}$ & $\begin{array}{c}\text { Non-NACT } \\
(\mathbf{n = 1 8})\end{array}$ & P Value \\
\hline Chemoradiation & $4(33.3)$ & $11(61.1)$ & 0.264 \\
Chemotherapy & $3(25)$ & $1(5.5)$ & 0.274 \\
Radiation & $1(8.3)$ & 0 & 0.400 \\
Patients without any adjuvant therapy & $4(33.3)$ & $6(33.3)$ & 1 \\
\hline
\end{tabular}

Note. NACT: Neoadjuvant chemotherapy.

was 31 (14-69) and 33 (12-43) months, respectively. In addition, 1 (8.3\%) patient in the NACT group and $3(16.6 \%)$ patients in the non-NACT group had a recurrence, which was not statistically significant. Finally, 2 (15.3\%) patients of the NACT group and 2 (11.1) other cases of non-NACT died, one of the patients in this group had died of a cause other than cervical cancer.

\section{Discussion}

This study compared radical hysterectomy in women with LACC receiving NACT with radical hysterectomy in women with early cervical cancer receiving NACT. The results showed that radical hysterectomy after NACT is safe. Post-operative blood transfusion was significantly higher in the NACT group compared to the non-NACT group, which was probably due to lower preoperative $\mathrm{Hb}$ in the NACT group. Operative time, EBL, intra-operative complications, and other post-operative complications were similar in both groups.

Despite the fact that chemoradiation has been recommended for women with LACC by the US-NCI since 1999 (10), NACT followed by radical surgery is a common practice in several countries outside the United States (11).

In a review article published in 2018, NACT followed by radical hysterectomy had no significant advantage compared with radical hysterectomy alone. More than $50 \%$ of operated patients required subsequent RT, which had significant morbidity and increased treatment cost without any significant benefit (1).

In another study, 151 patients with bulky IB cervical cancer entered the study, all received NACT and then underwent a radical hysterectomy. Post-operative chemotherapy was given for all patients regardless of the final pathology and $26.7 \%$ of them required adjuvant radiotherapy (9).

However, in our study, $4(33.3 \%)$ patients required chemoradiation and one (8.6\%) patient received radiotherapy.

In a study performed on 91 patients with stage IB2 cervical cancer, all cases received one of the following treatment modalities of chemoradiation, radical hysterectomy with or without adjuvant treatment, and NACT, followed by radical surgery with or without adjuvant. The 5-year overall survival in the NACT group with radical surgery, radical hysterectomy with or without

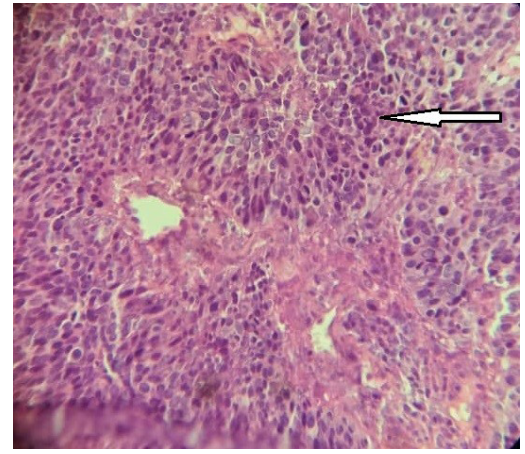

Figure 1. Invasive Squamous Cell Carcinoma in a Patient Prior to NACT Stained With Eosin and Hematoxylin (x400). Note. NACT: Neoadjuvant chemotherapy.

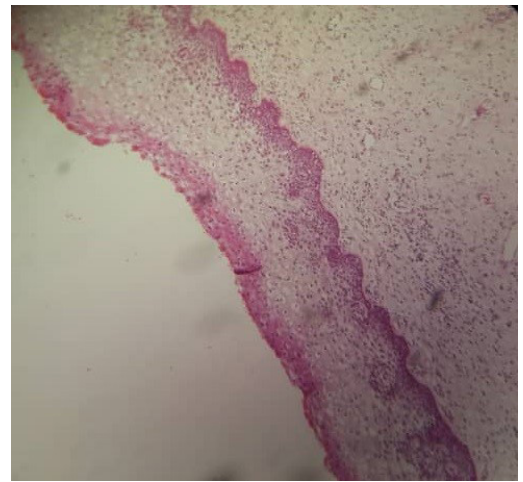

Figure 2. Tumor-free Pathology After NACT in the Patient Stained With Eosin and Hematoxylin (x250). Note. NACT: Neoadjuvant chemotherapy.

adjuvant therapy, and chemoradiation were 94.1, 93.8, and $80.1 \%$ although no significant differences were found in this regard (12).

In the study by Robova et al, all patients received NACT and then underwent radical hysterectomy. In other words, $18.3 \%$ of patients had positive pelvic lymph nodes and $17.2 \%$ of them experienced a relapse (9). In our study, one $(8.6 \%)$ patient from the NACT group had a recurrence and $3(23 \%)$ patients had positive pelvic lymph nodes.

In a study on 40 patients with stage IB-IIB cervical cancer, all cases underwent a laparoscopic radical hysterectomy after NACT, median operation time was 305 minutes (215430 ), and median EBL was $250 \mathrm{~mL}$ (100-400). Moreover, $10 \%$ of patients required blood transfusion after surgery, the median of the follow-up was 37 (10-69) months, and 3 (7.5\%) patients represented a relapse (13).

Likewise, robotic radical hysterectomy following NACT was compared with robotic radical hysterectomy alone in similar research. In other words, intra- and post-operative complications, patients' final pathology, EBL, duration of surgery, and length of hospital stay in both groups were compared in their study. Only operative time was significantly shorter in the non-NACT group while other parameters were not significantly different in the two 


\section{groups (14).}

In two other studies, robotic radical hysterectomy with NACT was compared with robotic radical hysterectomy, and intra- and post-operative complications were [( 0 vs. $0),(3$ vs. $5 \%)]$ and $[(12$ vs. $7 \%),(13$ vs. $10 \%)]$, respectively. Patients' final pathology, EBL, duration of surgery [median: (260 vs. 260 minutes), (209 vs. 215 minutes)] and the length of hospital stay [median: ( 4 vs. 4 days)] in both groups were not significantly different $(15,16)$.

Based on the report by Minig et al, the median followup was 31 months, $83 \%$ of patients were not relapsed, and $90 \%$ of them were alive (14). In our study, the median of follow-up was 26 months in the NACT group. More precisely, $91.7 \%$ of patients were free of recurrence and $84.6 \%$ of them were alive.

Our study compared two groups with different stages of the disease and different treatment strategies. As a result, post-operative blood transfusion was significantly higher in the NACT group, which was probably due to the lower $\mathrm{Hb}$ of these patients at the beginning of the operation. Nevertheless, there was no significant difference in EBL, duration of operation, complications during surgery, and other post-operative complications. These findings are similar to those of recent studies.

However, our study has some limitations, the most important of which are retrospective design, a small number of patients, and differences in stage and treatment strategies in the two included groups.

Accordingly, NACT followed by radical hysterectomy in women with LACC is safe and reduces the need for radiotherapy. These results need to be confirmed in studies with a larger patient sample.

\section{Authors' Contribution}

PMG, MS, MJ, ESOM, MV and VR: concept and design. MBS, AA, ME and MP: data collection and interpretation of the data. MBS: performing of the study and writing of the draft. All authors read and approved the study.

\section{Conflict of Interests}

Authors declare that they have no conflict of interests.

\section{Ethical Issues}

This study was approved by the ethical committee of Tabriz University of Medical Sciences (IR.TBZMED.REC.1399.720).

\section{Financial Support}

Women's Reproductive Health Research Center, Department of Obstetrics and Gynecology, Faculty of Medicine, Tabriz University of Medical Sciences, Tabriz, Iran.

\section{References}

1. de la Torre M. Neoadjuvant chemotherapy in woman with early or locally advanced cervical cancer. Rep Pract Oncol Radiother. 2018;23(6):528-532. doi:10.1016/j.rpor.2018.09.005

2. Salihi R, Leunen K, Van Limbergen E, Moerman P, Neven P, Vergote I. Neoadjuvant chemotherapy followed by large cone resection as fertility-sparing therapy in stage IB cervical cancer. Gynecol Oncol. 2015;139(3):447-451. doi:10.1016/j.ygyno.2015.05.043

3. Angioli R, Plotti F, Montera R, et al. Neoadjuvant chemotherapy plus radical surgery followed by chemotherapy in locally advanced cervical cancer. Gynecol Oncol. 2012;127(2):290-296. doi:10.1016/j.ygyno.2012.07.104

4. Bhatla N, Aoki D, Sharma DN, Sankaranarayanan R. Cancer of the cervix uteri. Int J Gynaecol Obstet. 2018;143 Suppl 2:22-36. doi:10.1002/ijgo.12611

5. Benedet JL, Pecorelli S, Ngan HY, et al. Staging classifications and clinical practice guidelines for gynaecological cancers. Int J Gynaecol Obstet. 2000;70:207-312.

6. Pecorelli S. Revised FIGO staging for carcinoma of the vulva, cervix, and endometrium. Int J Gynaecol Obstet. 2009;105(2):103104. doi:10.1016/j.ijgo.2009.02.012

7. Pecorelli S, Zigliani L, Odicino F. Revised FIGO staging for carcinoma of the cervix. Int J Gynaecol Obstet. 2009;105(2):107108. doi:10.1016/j.ijgo.2009.02.009

8. Jemal A, Bray F, Center MM, Ferlay J, Ward E, Forman D. Global cancer statistics. CA Cancer J Clin. 2011;61(2):69-90. doi:10.3322/ caac. 20107

9. Robova H, Rob L, Halaska MJ, et al. High-dose density neoadjuvant chemotherapy in bulky IB cervical cancer. Gynecol Oncol. 2013;128(1):49-53. doi:10.1016/j.ygyno.2012.10.002

10. $\mathrm{NCl}$ Issues Clinical Announcement on Cervical Cancer: Chemotherapy plus Radiation Improves Survival. NCI Press Office; 1999.

11. Ye Q, Yuan HX, Chen HL. Responsiveness of neoadjuvant chemotherapy before surgery predicts favorable prognosis for cervical cancer patients: a meta-analysis. J Cancer Res Clin Oncol. 2013;139(11):1887-1898. doi:10.1007/s00432-013-1509-y

12. Hsieh HY, Huang JW, Lu CH, Lin JC, Wang L. Definite chemoradiotherapy is a competent treatment option in FIGO stage IB2 cervical cancer compared with radical surgery +/- neoadjuvant chemotherapy. J Formos Med Assoc. 2019;118(1 Pt 1):99-108. doi:10.1016/j.jfma.2018.01.015

13. Vizza E, Pellegrino A, Milani R, et al. Total laparoscopic radical hysterectomy and pelvic lymphadenectomy in locally advanced stage IB2-IIB cervical cancer patients after neoadjuvant chemotherapy. Eur J Surg Oncol. 2011;37(4):364-369. doi:10.1016/j.ejso.2010.12.001

14. Minig L, Zanagnolo V, Cárdenas-Rebollo JM, Colombo N, Maggioni A. Feasibility of robotic radical hysterectomy after neoadjuvant chemotherapy in women with locally advanced cervical cancer. Eur J Surg Oncol. 2016;42(9):1372-1377. doi:10.1016/j.ejso.2016.04.060

15. Vitobello D, Siesto G, Pirovano C, leda N. Surgical outcomes of robotic radical hysterectomy after neoadjuvant chemotherapy for locally advanced cervical cancer: comparison with early stage disease. Eur J Surg Oncol. 2013;39(1):87-93. doi:10.1016/j. ejso.2012.10.001

16. Liu Z, Li X, Li W, Yang Y, Tao Y, Yao Y. Robotic nerve-sparing radical hysterectomy for locally advanced cervical cancer after neoadjuvant chemotherapy. Int J Gynaecol Obstet. 2015;131(2):152-155. doi:10.1016/j.ijgo.2015.05.017

\footnotetext{
(C) 2021 The Author(s); This is an open-access article distributed under the terms of the Creative Commons Attribution License (http:// creativecommons.org/licenses/by/4.0), which permits unrestricted use, distribution, and reproduction in any medium, provided the original work is properly cited.
} 